\title{
In vitro Analysis Provides New Insights into the Pharmacological Actions of Methanol Extract of Seeds of Tamarindus indica $\mathbf{L}$. and its Kupchan Fractions
}

\author{
Sharmeen Asad ${ }^{1}$, Farzana Kabir ${ }^{1}$, Safaet Alam ${ }^{1}$, Fahmida Tasnim Richi ${ }^{2}$, \\ Irin Pervin Anny ${ }^{1}$, Mst. Luthfun Nesa ${ }^{1}$ and Mohammad A. Rashid ${ }^{3}$ \\ ${ }^{1}$ Department of Pharmacy, State University of Bangladesh, Dhaka-1205, Bangladesh \\ ${ }^{2}$ Department of Pharmacy, Faculty of Pharmacy, University of Dhaka, Dhaka-1000, Bangladesh \\ ${ }^{3}$ Department of Pharmaceutical Chemistry, Faculty of Pharmacy, University of Dhaka, Dhaka-1000, \\ Bangladesh
}

(Received: July 19, 2021; Accepted: October 10, 2021; Published (web): January 29, 2022)

\begin{abstract}
Tamarind or Tamarindus indica L. is a multipurpose plant distributed throughout the tropics including Bangladesh. The present study was conducted to establish the preliminary antioxidant, antimicrobial, anti-inflammatory and thrombolytic activities of methanol extract of $T$. indica seeds along with its Kupchan fractions. To evaluate the antioxidant properties, the total phenolic content of $T$. indica was determined and expressed in gallic acid equivalent (GAE). Alongside, DPPH free radical scavenging assay was performed to ensure the antioxidant properties of the seeds where the methanolic crude extract revealed the maximum activity having $\mathrm{IC}_{50}$ value of $9.43 \mu \mathrm{g} / \mathrm{ml}$. In the antimicrobial assay by disk diffusion method, only non-polar fractions of the extract showed mild antimicrobial activity against the test organisms tested while the polar crude methanol extract exhibited the maximum inhibition (58.16\%, p $<0.001)$ of hypotonic solution-induced erythrocyte rupture in anti-inflammatory investigation among all the partitionates. During evaluation of thrombolytic activity in terms of percent of clot lysis, the methanol soluble fraction exhibited the highest percent of thrombolysis (23.5\%) as compared to the reference standard, streptokinase $(64.25 \%)$. The findings of the current study rationalize some of the traditional uses of $T$. indica and preliminarily ascertain its bioactive potential, which may act as a base for phytochemical and mechanism-based pharmacological studies of the plant in future.
\end{abstract}

Key words: Tamarindus indica, seed, antioxidant, antimicrobial, thrombolytic, membrane stabilizing activity.

\section{Introduction}

From the ancient era, medicinal plants assume a vital role in the security of human wellbeing. It has been accounted that $66 \%$ of the world's plant species contain medicinal properties (Krishnaiah et al., 2011). These medicinal plants contain various components of therapeutic properties so they can be utilized as drugs or formulations to treat different human illnesses (WHO, 1993). The possibility of the research work with these medicinal plants will not be fading out easily due to their natural abundance (Alam et al., 2020; Emon et al., 2020).
Tamarindus indica L. (family: Fabaceae, subfamily: Caesalpinioideae, Common name: Tamarind) is a tropical fruit with a plethora of nutritional and medicinal values which is locally known as "Tetul" (Kumar and Bhattacharya, 2008). Though it is indigenous to Africa, it has been naturalized and produced throughout Asia- Bangladesh, Indonesia, and Thailand (El-Siddig, 2006). Traditionally, it is used to heal wounds and treat fever, stomachache, colds and coughs, diarrhea along with as an antidote for snake bites (Buchholz and Melzig, 2016; Kaur

Corresponding author: Mohammad A. Rashid, E-mail: r.pchem@yahoo.comm

DOI: https://doi.org/10.3329/bpj.v25i1.57835 
and Bhullar, 2016). Previous pharmacological investigations of various parts i.e. fruit, leaves, bark, pulp and flowers have reported its role as antidiabetic, anti-inflammatory, antimicrobial and its potential role in the treatment or prevention of obesity and other chronic diseases (Bhadoriya et al., 2011; Reis et al., 2016). Abundant bioactive compounds such as tannins, polyphenols, and phenolic antioxidants ( $\beta$-sitosterol, eicosanoic acid, $n$-hexacosane, pinitol, and proanthocyanidins) also have been isolated from this plant (Landgraf Guiguer and Barbalho, 2016). Enriched in nutrients and chemical diversity, otherwise inedible and wasted seeds have been considered for their newfound usage as an inexpensive alternative protein source after detailed processing to remove tannins (Alam et al., 2021; El-Siddig, 2006; Siddhuraja et al., 1995). In continuation, to reduce the waste of tamarind seeds, we herein conducted in vitro assays on methanol extract of $T$. indica seeds to find out the logical evidence for its medicinal properties and explore its preliminary total phenolic content, DPPH free radical scavenging, antimicrobial, anti-inflammatory (membrane stabilizing) and thrombolytic potential.

\section{Materials and Methods}

Collection, drying, and extraction: T. indica seeds were collected in August of 2015 from Manikgonj district of Bangladesh, and identified by an expert taxonomist in Bangladesh National Herbarium, Dhaka. A voucher specimen was also recorded (DACB 36425) for this collection. The seeds were thoroughly cleaned before being sun dried for two weeks followed by grinding into a coarse powder. Approximately $300 \mathrm{~g}$ of dried ground seeds were steeped in $1.5 \mathrm{~L}$ of methanol for four weeks followed by filtering through cotton plug and Whatman No. 1 filter paper. The filtrate was concentrated at $45^{\circ} \mathrm{C}$ maintaining decreased pressure using a rotary evaporator. The petroleum ether (PESF), carbon tetrachloride (CTCSF), and aqueous soluble fractions (AQSF) were obtained from the concentrated methanol extract (ME, approximately $10 \mathrm{~g})$ using the modified Kupchan partitioning protocoll (VanWagenen et al., 1993). Thereafter, the pharmacological functions of the crude extract (ME) and its Kupchan fractions were studied individually.

Drugs and chemicals: All of the reagents employed in this research work, were of analytical grade and purchased from reputable vendors. Merck (Darmstadt, Germany) supplied the methanol, Tween-80, and ascorbic acid. Sigma Chemicals Co. (St. Louis, MO, USA) provided 1,1-diphenyl-2picryl-hydrazyl radical (DPPH) and Folin-Ciocalteu reagent (FCR) whereas, normal saline solution was purchased from Beximco Infusion Ltd., Bangladesh and acetylsalicylic acid and streptokinase were procured from Incepta Pharmaceuticals Ltd., Bangladesh during this research work.

Antioxidant activity analysis: The antioxidant properties of the extract were assessed using two distinct methods.

a) Total phenolics analysis: Considering FolinCiocalteu reagent as an oxidizing agent and gallic acid as a standard, the total phenolic content of $T$. indica extracts was determined (Harbertson and Spayd, 2006). In this experiment, $2.5 \mathrm{ml}$ of FolinCiocalteu reagent diluted 10 times with water, and 2 $\mathrm{ml}$ of sodium carbonate ( 7.5 percent $\mathrm{w} / \mathrm{v})$ solution were merged with $0.5 \mathrm{ml}$ of each extract $(2 \mathrm{mg} / \mathrm{ml})$ in water. After that, the mixtures were allowed to sit at room temperature for 20 minutes. Finally, with a UVvisible spectrophotometer, the absorbance was recorded at $760 \mathrm{~nm}$. Total phenolics were determined using a calibration curve created by measuring the standard gallic acid values $(0-100 \mathrm{~g} / \mathrm{ml})$ and reported as mg of GAE (gallic acid equivalent)/gm of dried extract.

b) Determination of free radical scavenging activity: The antioxidant activity of the methanol crude extract of $T$. indica and its Kupchan fractions on the stable radical 1,1- diphenyl-2-picrylhydrazyl (DPPH) was assessed using ascorbic acid as a positive control (Brand-Williams et al., 1995). With a successive serial dilution method, $2.0 \mathrm{mg}$ of each extract was dissolved in methanol, producing solutions with different concentrations of 500, 250, $125,62.50,31.25,15.62,7.81,3.91,1.95$, and 0.98 
$\mathrm{g} / \mathrm{ml}$. The extracts were then combined with $3.0 \mathrm{ml}$ of a DPPH-methanol solution $(20 \mathrm{~g} / \mathrm{ml})$ and allowed to stand for 20 minutes. Finally, the absorbance was measured at $517 \mathrm{~nm}$ and from these values, the corresponding percentage of inhibition was determined utilizing the following equation:

$$
\% \text { Inhibition }=\left\{\left(\mathrm{A}_{0}-\mathrm{A}_{1}\right) / \mathrm{A}_{0}\right\} \times 100
$$

Where, $\mathrm{A}_{0}=$ the absorbance of control; $\mathrm{A}_{1}=$ the absorbance of sample

Then $\%$ inhibitions were plotted against respective concentrations used and from the graph, the $\mathrm{IC}_{50}$ was calculated.

Antimicrobial activity analysis: The disc diffusion technique was employed to investigate the antibacterial activity of methanol crude extract of $T$. indica and its Kupchan fractions (Bauer, 1966) against 5 gram-positive bacteria, 8 gram-negative bacteria and 3 fungi utilizing nutrient agar medium. The extracts were carefully put on the agar plates in the previously designated zones. Positive controls of standard ciprofloxacin and fluconazole discs (30 $\mathrm{g} /$ discs) were utilized to confirm activity against test organisms. The zones of inhibition developed by the extracts were analyzed and correlated with the standard.

Membrane stabilizing activity analysis: Hypotonic-induced hemolysis was studied by employing previously mentioned method with slight modification to evaluate the membrane stabilizing activity of the methanol extract and its fractions (Shinde et al., 1999). For this $5 \mathrm{ml}$ of whole blood from healthy human volunteers was taken into a tube containing $2.2 \mathrm{mg}$ di-potassium salt of EDTA per 1.0 $\mathrm{ml}$ of blood for the preparation of human red blood cell suspension. Then, the collected blood was centrifuged at $3000 \mathrm{rpm}$ for 10 minutes. After that, the supernatant was separated and the blood cells were washed with saline in $10 \mathrm{mM}$ sodium phosphate buffer ( $\mathrm{pH}$ 7.4) for three times using the same volume as the supernatant followed by reconstitution in the same volume of this isotonic buffer solution. After that, $5 \mathrm{ml}$ of hypotonic solution $(50 \mathrm{mM} \mathrm{NaCl})$ in $10 \mathrm{mM}$ sodium phosphate buffered saline ( $\mathrm{pH} 7.4$ ) containing either reference drug, acetyl salicylic acid
$(0.1 \mathrm{mg} / \mathrm{ml})$ or the extract $(1 \mathrm{mg} / \mathrm{ml})$, was combined with $0.5 \mathrm{ml}$ of the resultant solution. The control sample was composed of $0.5 \mathrm{ml}$ of RBCs which was combined with hypotonic buffered saline. Then, the mixture was incubated at room temperature for 10 minutes before being centrifuged at $3000 \mathrm{rpm}$ for 10 minutes and the optical density (OD) of the supernatant was measured at $540 \mathrm{~nm}$. The percentage inhibition of hemolysis was computed by using the equation as followed-

$\%$ inhibition of hemolysis $=\left\{\left(\mathrm{OD}_{\text {control }}-\mathrm{OD}_{\text {test sample }}\right) /\right.$

$$
\left.\mathrm{OD}_{\text {control }}\right\} \times 100
$$

Thrombolytic activity analysis: For the thrombolytic test, $6 \mathrm{ml}$ of venous blood was taken from healthy donors and placed in 10 separate preweighed sterile vials ( $1 \mathrm{ml} /$ tube) (Prasad et al., 2006). Then the vials were kept in an incubator at $37^{\circ} \mathrm{C}$ for 45 minutes. After the clot had been formed, the serum was fully withdrawn without agitating it and each vial was weighed for the determination of the clot weight (clot weight $=$ weight of clot containing tube - weight of tube alone). Each of the extracts (1 $\mathrm{mg} / 100 \mu \mathrm{l}$ water) was then poured into each vial holding the known weighed clot in the following step. In this study, $100 \mu \mathrm{l}$ of streptokinase and $100 \mu 1$ of distilled water were utilized as the positive and negative control, respectively. After that, all of the vials were incubated at $37^{\circ} \mathrm{C}$ for 90 minutes to check for clot lysis. The released fluid was collected after incubation and the vials were further weighed to monitor the difference in weight after the clot was disrupted. As demonstrated below, the alteration in weight acquired before and after clot lysis was represented as a percentage of clot lysis.

$\%$ of thrombolysis $=$ (weight of clot after treatment $/$ weight of clot before treatment $) \times 100$

Statistical analysis: For each experiment, three replicates of each sample were utilized, and statistical analysis was executed by using the Student's t-test. The mean and standard deviation have been used to present all data. 


\section{Results and Discussion}

Antioxidant activity analysis: The antioxidant properties of the test sample have been summarized in the Table 1.

a) Total phenolic content: In this study, the highest content of phenolic (27.87 mg of GAE/gm) was evident by the methanol extract of $T$. indica seeds (Table 1). The phenolic content of other fractions was observed in a range of $7-17 \mathrm{mg}$ of GAE/gm signifying mild to moderate range of antioxidant potentials.

b) DPPH free radical scavenging assay: In case of DPPH free radical scavenging activity analysis, the methanol extract exhibited the highest scavenging of $\mathrm{IC}_{50}=9.43 \mu \mathrm{g} / \mathrm{ml}$ which was followed by the carbon tetrachloride fraction with $\mathrm{IC}_{50}=22.25 \mu \mathrm{g} / \mathrm{ml}$ (Table 1). Here, this study was compared with the standard ascorbic acid with the $\mathrm{IC}_{50}=3.05 \mu \mathrm{g} / \mathrm{ml}$. As, phenolic acids and flavonoids are reported to exhibit prominent antioxidant potential (Emon et al., 2021; Rudra et al., 2020; Shahidi et al., 1992), the free radical neutralizing property of the methanol extract might be due to the said phytoconstituents which was further justified by the highest presence of phenolic content in the same fractionate.

Table 1. Total phenolic content, free radical scavenging, membrane stabilizing (\% inhibition of hypotonic solution induced hemolysis) and thrombolytic activities of $T$. indica whole seeds.

\begin{tabular}{|c|c|c|c|c|c|}
\hline \multirow{2}{*}{$\begin{array}{l}\text { Sample/ } \\
\text { standard }\end{array}$} & \multirow{2}{*}{$\begin{array}{l}\text { Total phenolic } \\
\text { content (mg of } \\
\text { GAE/gm of } \\
\text { extract) }\end{array}$} & \multirow{2}{*}{$\begin{array}{l}\text { DPPH Free radical } \\
\text { scavenging activity } \\
\quad\left(\mathrm{IC}_{50} \mu \mathrm{g} / \mathrm{ml}\right)\end{array}$} & \multicolumn{2}{|c|}{$\begin{array}{l}\text { Membrane stabilizing activity } \\
\text { ( Hypotonic solution induced) }\end{array}$} & \multirow{2}{*}{$\begin{array}{c}\text { Thrombolytic (\% clot } \\
\text { lysis) }\end{array}$} \\
\hline & & & Mean absorbance & Inhibition (\%) & \\
\hline METI & $27.87 \pm 0.12$ & $9.43 \pm 0.22 * *$ & $0.89 \pm 0.01$ & $58.16 \pm 0.33 * *$ & $23.5 \pm 0.49 * *$ \\
\hline PESF & $15.03 \pm 0.10$ & $39.15 \pm 0.34 * *$ & $1.65 \pm 0.04$ & $22.38 \pm 1.09 * *$ & $2.49 \pm 0.27 * *$ \\
\hline CTCSF & $17.31 \pm 0.22$ & $22.25 \pm 0.28 * *$ & $1.92 \pm 0.01$ & $10.01 \pm 0.30 * *$ & $22.51 \pm 0.31 *$ \\
\hline AQSF & $7.38 \pm 0.19$ & $77.31 \pm 0.29 * *$ & $1.03 \pm 0.03$ & $51.63 \pm 0.77 * *$ & $17.26 \pm 0.29 * *$ \\
\hline $\mathrm{AA}$ & & $3.05 \pm 0.15^{* *}$ & & & \\
\hline ASA & & & $0.60 \pm 0.02$ & $71.89 \pm 0.29 * *$ & \\
\hline SK & & & & & $64.25 \pm 0.36 * *$ \\
\hline Blank & -- & -- & $2.13 \pm 0.08$ & -- & $10.47 \pm 0.38$ \\
\hline
\end{tabular}

Here, METI: Methanolic crude extract of $T$. indica seeds, PESF: Pet ether soluble fraction, CTCSF: Carbon tetrachloride fraction, AQSF: Aqueous soluble fraction, AA = Ascorbic acid, ASA: Acetyl salicylic acid, SK: Streptokinase. All values are presented as mean \pm standard deviation. ${ }^{*} p<0.005,{ }^{*} p<0.001$ is considered as statistically significant as compared to negative control.

Antimicrobial activity analysis: In the probing of antimicrobial activity by disc diffusion assay, only pet ether soluble fraction and carbon tetrachloride fraction of seeds of $T$. indica exhibited a smaller extent of antimicrobial activity (zone of inhibition = $7-11 \mathrm{~mm}$ ) against the test organisms at concentrations of $400 \mu \mathrm{g} / \mathrm{ml}$ (Table 2). It can be summarized that the non-polar fractions (pet ether and carbon tetrachloride) were observed with more activity against the test strains as compared to the polar fractions (methanol and water).
Membrane stabilizing activity analysis: In table 1 , the percentage of erythrocyte membrane rupture inhibition of different fractionates of the seed of $T$. indica has been presented. The highest RBC membrane protecting capacity was exhibited for the methanol extract of seeds of $T$. indica $(58.16 \%)$ when compared with the standard acetylsalicylic acid (71.89\%). The second highest membrane stabilizing activity was noticed by the aqueous soluble fraction (51.63\%) whereas mild erythrocyte membrane protecting capacity was indicated by the pet ether soluble fraction $(22.38 \%)$ and carbon tetrachloride 
fraction (10.01\%). Stabilization of erythrocyte membrane has long been extrapolated to stipulate the effects of different compounds on the stabilization of lysosomal membrane that is known to be related to the release of inflammatory cytokines (Omale and Okafor, 2008; Shinde et al., 1999). Based on this results, it can be proposed that the more polar fractions were noticed with more ability to resist the cell lysis as compared to the non-polar pet ether and carbon tetrachloride fraction which is suggestive of the presence of constituents with therapeutic potentials for inflammatory conditions.

Table 2. Antimicrobial activity of different fractions of seeds of $T$. indica.

\begin{tabular}{lcccccc}
\hline \multicolumn{1}{c}{ Test Microorganisms } & \multicolumn{5}{c}{ Diameter of Zone of Inhibition (mm) } \\
\cline { 2 - 6 } Gram Positive Bacteria & METI & PESF & CTCSF & DCMSF & AQSF & Ciprofloxacin \\
Bacillus sereus & - & 8 & 8 & - & - & 40 \\
B. megaterium & - & 9 & - & - & - & 42 \\
B. subtilis & - & 7 & - & - & - & 41 \\
Staphylococcus aureus & - & 10 & 8 & - & - & 45 \\
Sarcina lutea & - & 10 & 9 & - & - & 46 \\
Gram Negative Bacteria & & & & & \\
Escherichia coli & - & 7 & 8 & - & - & 45 \\
Pseudomonas aureus & - & 10 & 8 & - & - & 41 \\
Salmonella paratyphi & - & - & 7 & - & - & 35 \\
S. typhi & - & - & - & - & - & 45 \\
Shigella boydii & - & 8 & 8 & - & - & 42 \\
Sh. dysenteriae & - & 7 & 7 & - & - & 41 \\
Vibrio mimicus & - & 10 & 9 & - & - & 38 \\
V. parahemolyticus & - & 11 & 7 & - & - & 44 \\
\hline Fungi & & & & & & Fluconazole \\
\hline Aspergillus niger & - & 7 & 10 & - & - & 47 \\
Candida albicans & - & - & - & - & - & 42 \\
Sacharomyces cerevacae & - & 7 & 7 & - & - & 46 \\
\hline
\end{tabular}

Thrombolytic activity analysis: The percentages of clot lysis of different fractions of the seed of $T$. indica have been illustrated in Table 1. The highest thrombolytic activity (23.5\%) was evident by the methanol extract of seeds of $T$. indica whereas the standard streptokinase showed $64.25 \%$ thrombolysis. On the other hand, moderate clot lysis ability was also noticed by carbon tetrachloride fraction $(22.51 \%)$ and aqueous soluble fraction (17.26\%). However, the very weak thrombolytic activity was observed by the pet ether soluble fraction $(2.49 \%)$. The current result is suggestive of the probable availability of thrombolytic agents in the polar fractions.

\section{Conclusion}

The methanol extract of seeds of $T$. indica and its Kupchan fractions were investigated for antioxidant, antimicrobial, anti-inflammatory and thrombolytic activities. After performing the evaluations, it may be assumed that these extractives can be considered as a potential source of antioxidant, anti-inflammatory, and thrombolytic therapeutic agents. Additional comprehensive investigations are recommended to isolate the bioactive components from this plant part 
responsible for the exerted pharmacological actions and to know their in-depth underlying mechanisms.

\section{Declaration}

All authors read the manuscript and approve it for the publication and no part of this manuscript has been published before in any journal.

\section{Conflict of interest}

The authors state that they have no conflict of interest.

\section{Acknowledgements}

The authors are thankful to the authorities of Department of Pharmacy, State University of Bangladesh for providing necessary facilities to carry out this research work.

\section{References}

Alam, S., Emon, N.U., Shahriar, S., Richi, F.T., Haque, M.R., Islam, M.N. and Sakib, S.A., Ganguly, A. 2020. Pharmacological and computer-aided studies provide new insights into Millettia peguensis Ali (Fabaceae). Saudi Pharm. J. 28, 1777-1790.

Alam, S., Rashid, M.A., Sarker, M.M.R., Emon, N.U., Arman, M., Mohamed, I.N. and Haque, M.R. 2021. Antidiarrheal, antimicrobial and antioxidant potentials of methanol extract of Colocasia gigantea Hook.f. leaves: evidenced from in vivo and in vitro studies along with computer-aided approaches. BMC Сотр. Med. Therap. 21, 1-12.

Bauer, A.W. 1966. Antibiotic susceptibility testing by a standardized single disc method. Am. J. Clin. Pathol. 45, 149-158.

Bhadoriya, S.S., Ganeshpurkar, A., Narwaria, J., Rai, G. and Jain, A.P. 2011. Tamarindus indica: extent of explored potential. Pharmacogn. Rev. 5, 73.

Brand-Williams, W., Cuvelier, M.-E. and Berset, C. 1995. Use of a free radical method to evaluate antioxidant activity. LWT-Food Sci. Tech. 28, 25-30.

Buchholz, T. and Melzig, M.F. 2016. Medicinal plants traditionally used for treatment of obesity and diabetes mellitus-screening for pancreatic lipase and $\alpha$-amylase inhibition. Phytother. Res. 30, 260-266.

El-Siddig, K. 2006. Tamarind: Tamarindus Indica L. Crops for the Future.
Emon, N.U., Alam, S., Rudra, S., Al Haidar, I.K., Farhad, M., Rana, M.E.H. and Ganguly, A. 2021. Antipyretic activity of Caesalpinia digyna (Rottl.) leaves extract along with phytoconstituent's binding affinity to COX-1, COX-2 and mPGES-1 receptors: In vivo and in silico approaches. Saudi J. Biol. Sci. 28, 5302.

Emon, N.U., Alam, S., Rudra, S., Chowdhury, S., Rajbangshi, J.C. and Ganguly, A. 2020. Evaluation of pharmacological potentials of the aerial part of Achyranthes aspera L.: in vivo, in vitro and in silico approaches. Adv. Tradit. Med.1-14.

Harbertson, J.F. and Spayd, S. 2006. Measuring phenolics in the winery. Am. J. Enol. Vitic. 57, 280-288.

Kaur, M. and Bhullar, G.K. 2016. Partial characterization of Tamarind (Tamarindus indica L.) kernel starch oxidized at different levels of sodium hypochlorite. Int. J. Food Prop. 19, 605-617.

Krishnaiah, D., Sarbatly, R. and Nithyanandam, R. 2011. A review of the antioxidant potential of medicinal plant species. Food Bioprod. Process. 89, 217-233.

Kumar, C.S. and Bhattacharya, S. 2008. Tamarind seed: properties, processing and utilization. Crit. Rev. Food Sci. Nutr. 48, 1-20.

Landgraf Guiguer, E. and Barbalho, S. 2016. Tamarindus indica $\mathrm{L}$. A plant with multiple medicinal purposes. $J$. Pharmacogn. Phytochem. 5, 50-54.

Omale, J. and Okafor, P.N. 2008. Comparative antioxidant capacity, membrane stabilization, polyphenol composition and cytotoxicity of the leaf and stem of Cissus multistriata. Afr. J. Biotechnol. 7.

Prasad, S., Kashyap, R.S., Deopujari, J.Y., Purohit, H.J., Taori, G.M. and Daginawala, H.F., 2006. Development of an in vitro model to study clot lysis activity of thrombolytic drugs. Thromb. J. 4, 14.

Reis, P.M.C.L., Dariva, C., Vieira, G.Â.B. and Hense, H. 2016. Extraction and evaluation of antioxidant potential of the extracts obtained from tamarind seeds (Tamarindus indica), sweet variety. J. Food Eng. 173, 116-123.

Rudra, S., Sawon, M.S.U., Emon, N.U., Alam, S., Tareq, S.M., Islam, M.N. and Shakil, M. 2020. Biological investigations of the methanol extract of Tetrastigma leucostaphylum (Dennst.) Alston ex Mabb. (Vitaceae): In vivo and in vitro approach. J. Adv. Biotechnol. Exp. Ther. 3, 216-224.

Shahidi, F., Janitha, P.K. and Wanasundara, P.D., 1992. Phenolic antioxidants. Crit. Rev. Food Sci. Nutr. 32, 67-103. 
Shinde, U.A., Phadke, A.S., Nair, A.M., Mungantiwar, A.A., Dikshit, V.J. and Saraf, M.N. 1999. Membrane stabilizing activity-a possible mechanism of action for the anti-inflammatory activity of Cedrus deodara wood oil. Fitoterapia 70, 251-257.

Siddhuraja, P., Vijayakumari, K. and Janardhanan, K. 1995. Nutritional and Antinutritional Properties of the Underexploited Legumes Cassia laevigata Willd. and Tamarindus indica L. J. Food Comp. Anal. 8, 351362.
VanWagenen, B.C., Larsen, R., Cardellina, J.H., Randazzo, D., Lidert, Z.C., Swithenbank, C. 1993. Ulosantoin, a potent insecticide from the sponge Ulosa ruetzleri. J. Org. Chem. 58, 335-337.

WHO, 1993. Research guidelines for evaluating the safety and efficacy of herbal medicines. Manila: WHO Regional Office for the Western Pacific. 